\title{
Cefaleia por uso excessivo de medicamentos entre os estudantes de medicina
}

\author{
Medication overuse headache among the medical students
}

Cefalea por uso excesivo de medicamentos entre los estudiantes de medicina

Eva Gabryelle Vanderlei Carneiro ${ }^{1 *}$, Maria Gabriela Rocha Melo ${ }^{1}$, Laércio Pol Fachin², Mariana Cota Bastos $^{3}$.

\section{RESUMO}

Objetivo: Avaliar o desenvolvimento da cefaleia por uso excessivo de medicamentos (CEM) nos discentes de medicina, bem como determinar os possíveis gatilhos. Métodos: Pesquisa quantitativa, do tipo exploratória, utilizando um questionário aplicado aos discentes de medicina de um centro universitário. Resultados: Participaram da pesquisa 240 discentes de medicina, 23,50\% ( $n=43)$ preenchendo os critérios para cefaleia crônica. Destes, $26(60,47 \%)$ apresentam critérios para CEM. A prevalência de cefaleia crônica foi uma variável dependente do consumo de analgésicos simples $(p<0,0001)$, analgésicos combinados $(p<$ $0,0001)$, ergots $(p<0,0001)$ e triptanos $(p=0,0450)$. Dos gatilhos investigados, $51,25 \%$ dos discentes referiram não ter uma qualidade do sono excelente e $37,92 \%$ associaram ao período de provas/apresentação de trabalhos $(p<0,0001)$. Apenas $10,83 \%$ se consideraram etilistas e $1,67 \%$, tabagistas. Dentre os fatores protetores, apenas $23,26 \%$ realizavam terapia não medicamentosa (acupuntura, compressa gelada) e $51,16 \%$ praticavam atividade física regularmente. Conclusão: Os achados do estudo alertam as comunidades científicas sobre os riscos da CEM entre os discentes de medicina. Práticas de conscientização e estratégias pedagógicas devem ser instituídas em todas a $\mathrm{m} \mathrm{s}$ instituições de ensino superior a fim de minimizar os prejuízos acadêmicos.

Palavras-chave: Cefaleia, Automedicação, Estudantes de medicina.

\begin{abstract}
Objective: To evaluate the development of medication overuse headache $(\mathrm{MOH})$ in medical students, as well as to determine possible triggers. Methods: Quantitative research, exploratory, using a questionnaire applied to medical students at a university center. Results: 240 medical students participated in the research, $23.50 \%$ $(n=43)$ fulfilling the criteria for chronic headache. Of these, $26(60.47 \%)$ have criteria for $\mathrm{MOH}$. The prevalence of chronic headache was a variable dependent on the consumption of simple analgesics $(p<0.0001)$, combined analgesics $(p<0.0001)$, ergots $(p<0.0001)$ and triptans $(p=0.0450)$. Of the investigated triggers, $51.25 \%$ of the students reported not having an excellent sleep quality and $37.92 \%$ associated it with the period of tests/presentation of papers ( $p<0.0001$ ). Only $10.83 \%$ considered themselves alcoholics and $1.67 \%$ smokers. Among the protective factors, only $23.26 \%$ underwent non-drug therapy (acupuncture, cold compress) and $51.16 \%$ practiced physical activity regularly. Conclusion: The study's findings alert scientific communities to the risks of $\mathrm{MOH}$ among medical students. Awareness-raising practices and pedagogical strategies must be instituted in all higher education institutions in order to minimize academic losses.
\end{abstract}

Keywords: Headache, Self-medication, Medical students.

${ }^{1}$ Centro Universitário Cesmac (CESMAC), Maceió - AL. *E-mail: evagvc96@gmail.com

2 Universidade Federal do Rio Grande do Sul (UFRGS), Porto Alegre - RS.

${ }^{3}$ Universidade Federal de Alagoas (UFAL), Maceió - AL. 


\section{RESUMEN}

Objetivo: Evaluar el desarrollo de cefalea por uso excesivo de medicamentos (CEM) en estudiantes de medicina, así como determinar posibles desencadenantes. Métodos: Investigación cuantitativa, exploratoria, mediante cuestionario aplicado a estudiantes de medicina de un centro universitario. Resultados: 240 estudiantes de medicina participaron en la investigación, el 23,50\% $(n=43)$ cumpliendo los criterios de cefalea crónica. De estos, $26(60,47 \%)$ tienen criterios para EMF. La prevalencia de cefalea crónica fue una variable dependiente del consumo de analgésicos simples $(p<0,0001)$, analgésicos combinados $(p<0,0001)$, ergots $(p<0,0001)$ y triptanos $(p=0,0450)$. De los desencadenantes investigados, el $51,25 \%$ de los estudiantes refirió no tener una excelente calidad del sueño y el 37,92\% lo asoció con el período de pruebas/presentación de trabajos $(p<0,0001)$. Solo el $10,83 \%$ se consideraba alcohólico y el $1,67 \%$ fumador. Entre los factores protectores, solo el $23,26 \%$ se sometió a terapia no farmacológica (acupuntura, compresas frías) y el 51,16\% practicó actividad física con regularidad. Conclusión: Los hallazgos del estudio alertan a las comunidades científicas sobre los riesgos de la CEM entre los estudiantes de medicina. Se deben instituir prácticas de sensibilización y estrategias pedagógicas en todas las instituciones de educación superior para minimizar las pérdidas académicas.

Palabras clave: Cefalea, Automedicación, Estudiantes de medicina.

\section{INTRODUÇÃO}

A cefaleia acomete cerca de três bilhões de indivíduos no mundo, sendo altamente prevalente e com impacto negativo na qualidade de vida da população, é, por isso, considerada um relevante problema de saúde pública (STOVNER LJ, et al., 2018). No entanto, essa comorbidade permanece subdiagnosticada e subtratada em alguns grupos populacionais, especialmente nos estudantes universitários (BIRRU EM, et al., 2016).

Ao longo dos anos, várias pesquisas epidemiológicas procuraram avaliar a frequência e o impacto da cefaleia entre os discentes, principalmente no curso de medicina, já que estes são mais susceptíveis a vários fatores de estresse físico e psicológico (NOOR T, et al., 2016). Uma alta prevalência de cefaleia ao longo da graduação do curso médico foi encontrada em grupos de pesquisa em todo o mundo, variando de $53 \%$ a 98,8\% (DELEU D, et al., 2001; BIRRU EM, et al., 2016).

Sabe-se que, nos discentes universitários, a cefaleia está relacionada a dias perdidos de estudo e pior desempenho acadêmico (BIRRU EM, et al., 2016). Ademais, o estudo de Diniz BS, et al. (2011), realizado no Brasil, constatou que os alunos de medicina portadores de migrânea foram mais propensos ao baixo rendimento, demonstrado através do número de provas suplementares, absenteísmo escolar e prejuízo em atividades sociais.

Diante do exposto, esse grupo é classificado como potencialmente em risco para o desenvolvimento de cefaleia primária e mais suscetível à prática da automedicação (ALMEIDA CM, et al., 2015). A elevada prática de automedicação sem prescrição e sem orientação médica foi observada em até $96 \%$ dos grupos analisados por estudos realizados em diferentes universidades médicas (NOOR T, et al., 2016; ALSHOGRAN OY, et al., 2018), sendo a cefaleia a principal causa (IBRAHIM NK, et al., 2017; ALSHOGRAN OY, et al., 2018).

Dessa maneira, evidencia-se que o fácil acesso aos recursos de informações sobre os medicamentos, tal como a familiaridade com estes entre os discentes do curso médico (ABDI A, et al., 2018) contribui para este resultado. Os medicamentos geralmente são obtidos em casa com os familiares, na farmácia ou entre os próprios alunos na faculdade (LUKOVIC JA, et al., 2014).

Essa utilização indiscriminada de medicamentos analgésicos, principalmente, tem gerado uma preocupação crescente no cenário mundial devido às repercussões negativas que o uso crônico acarreta para os indivíduos (ABAY SM e AMELO W, 2010), como tratamento inadequado e uso excessivo (ALMEIDA CM, et al., 2015; BIRRU EM, et al., 2016). 
Entretanto, sabe-se que todas as medicações usadas para o tratamento sintomático da cefaleia podem causar a cefaleia por uso excessivo de medicamentos (CEM) (LAI JTF, et al., 2014). Sendo esta, uma complicação cada vez mais frequente e pouco conhecida na população, com prevalência mundial de $1 \%$ a 2\% (WESTERGAARD ML, et al., 2016).

De acordo com a terceira edição da International Classification of Headache Disorders (ICHD-3, 2018), a CEM ocorre em pacientes com cefaleia primária, que desenvolvem um novo tipo de cefaleia ou uma piora significativa na cefaleia pré-existente devido ao uso excessivo de medicamentos.

É característica a presença da dor em 15 ou mais dias no mês como consequência do uso excessivo de medicação para cefaleia aguda (em 10 ou mais ou 15 ou mais dias por mês, dependendo da classe medicamentosa) por mais de três meses.

Entendendo que os discentes de medicina apresentam uma elevada frequência de cefaleia primária e de automedicação, o propósito desta pesquisa foi avaliar o risco de CEM, bem como os possíveis fatores de risco e protetores para o desenvolvimento do quadro. Os resultados obtidos poderão contribuir na definição de estratégias para minimizar as complicações associadas ao tratamento inadequado das cefaleias primárias.

\section{MÉTODOS}

Trata-se de um estudo exploratório de natureza quantitativa realizado em um Centro Universitário Privado no estado de Alagoas. Foram convidados para o estudo um total de 823 discentes do curso de medicina, regularmente matriculados do $1^{\circ}$ ao $8^{\circ}$ período. Aqueles do período do internato ( $9^{\circ}$ ao $12^{\circ}$ período) foram excluídos do estudo por estarem em atividades fora da instituição, o que dificultava o acesso. $O$ período de coleta dos dados foi de fevereiro a dezembro de 2019.

A amostragem prevista para o estudo foi de 240 acadêmicos, sendo 30 participantes de cada período. Para realizar o cálculo do tamanho da amostra, utilizou-se o software $G^{\star} P o w e r ~ 3.1 .9 .2$. Como o plano de análise dos dados e o desenho da pesquisa é prever a comparação entre 10 amostras independentes, utilizouse como referência para o cálculo o teste ANOVA one-way.

Para determinar o tamanho da amostra, adotou-se três critérios: nível de significância de 0,05, tamanho do efeito de aproximadamente 0,25 e poder do teste a ser alcançado de 0,80 . Para atingir o tamanho de poder de $80 \%$, é demandado um nível de significância de 0,05 , com tamanho de efeito moderado $(\approx 0,25)$ e uma amostra de 240 indivíduos. A amostra é não probabilística, isto é, de conveniência, tendo a participação de discentes que, convidados, aceitaram colaborar voluntariamente.

Foi elaborado pelos pesquisadores um questionário impresso desenvolvido com 20 (vinte) assertivas construídas com a escala do tipo Likert. As assertivas foram elaboradas de forma breve e objetiva, em primeira pessoa, com vocabulário comum para fácil interpretação.

Tendo como embasamento a literatura científica mundial (LUKOVIC JA, et al 2014; LAI JTF, et al 2014; ICHD-3, 2018) foram criadas assertivas que contemplassem os aspectos relevantes para o estudo do tema: critérios para cefaleia crônica; critérios para cefaleia por uso excessivo de medicamentos de acordo com a classe medicamentosa; risco de automedicação; principais gatilhos para cefaleia entre estudantes; fatores protetores e de risco para o uso excessivo de medicamentos; e conhecimento sobre as complicações do uso excessivo de medicamentos.

As respostas dos questionários foram codificadas por meio do padrão de concordância e discordância, sendo transformadas em percentual. Para a análise estatística foram utilizados testes estatísticos (quiquadrado de Pearson, correlação de Pearson e de Spearman) para avaliar o nível de significância dos resultados encontrados.

Dessa forma, foram consideradas as variáveis: período do curso, frequência da cefaleia, prevalência de cefaleia por uso excessivo, insônia, prática de atividade física, tabagismo, etilismo, automedicação, conhecimento dos efeitos colaterais, excesso de medicamentos em uma das classes investigadas (analgésicos simples, anti-inflamatórios, triptanos, derivados do ergot, combinações de analgésicos ou opioides). 
Com a finalidade de auxiliar a análise dos dados, as assertivas elaboradas foram agrupadas em seis categorias pré-definidas (a priori), utilizando a metodologia de Bardin L (1977): critérios para cefaleia crônica, uso excessivo de medicamento por classe farmacológica, risco para automedicação, gatilhos para cefaleia, fatores protetores para o uso excessivo de medicamentos e conhecimento das complicações do uso excessivo medicamentos (Tabela 1).

Tabela 1 - Categorias de análise a priori elaboradas pelos autores com suas assertivas correspondentes no questionário.

\begin{tabular}{cc} 
Categorias de análise & Assertivas \\
\hline Critério para cefaleia crônica & 1 \\
Uso excessivo de medicamento por classe farmacológica & $2,3,4,5,6,7$ \\
Risco para automedicação & $8,9,10,11$ \\
Gatilhos para cefaleia & $12,15,16,17,18$ \\
Fatores protetores para o uso excessivo de medicamentos & 13,14 \\
Conhecimento das complicações dos medicamentos & 19,20 \\
\hline
\end{tabular}

Fonte: CARNEIRO EGV, et al., 2020.

O estudo foi aprovado pelo parecer $n^{\circ} 3.227 .703$ do Comitê de Ética em Pesquisa do Centro de Estudos Superiores de Maceió (CESMAC/CENTRO), inscrito sob CAAE 06778918.0.0000.0039. O trabalho atendeu às exigências da Resolução CNS n 466/2012 e n 510/2016. Todos os participantes assinaram o termo de consentimento livre e esclarecido antes de sua inclusão no estudo.

\section{RESULTADOS}

O estudo alcançou a amostragem prevista de 240 participantes. O perfil da amostra foi de 135 (56,25\%) do sexo feminino e $71(29,58 \%)$ do sexo masculino, $34(14,16 \%)$ discentes não desejaram responder o gênero. A idade variou entre 18 e 42 anos, média de 25 anos (desvio padrão $=9,12$ ). Os resultados da pesquisa serão apresentados de acordo com as categorias de análise a priori (Quadro 1).

Foi analisada a existência de características que preencham os critérios diagnósticos para cefaleia crônica entre os discentes de medicina, de acordo com a International Classification of Headache Disorder (ICHD-3, 2018). Assim, os discentes tinham que responder o grau de concordância com a assertiva "nos últimos três meses tive 15 ou mais dias de dor de cabeça por mês".

A maioria dos discentes, 166 (69,17\%), discordou da assertiva, sugerindo não apresentar características para cefaleia crônica. Entretanto, $43(23,50 \%)$ concordaram, totalmente ou parcialmente, com a assertiva. Destes, $24(55,81 \%)$ estavam nos períodos iniciais $\left(1^{\circ}-4^{\circ}\right)$ e $19(44,19 \%)$ nos períodos mais avançados $\left(5^{\circ}\right.$ - 8º). Houve uma predominância do sexo feminino, 33 (76,74\%), entre os discentes que concordaram com a assertiva. A estimativa da correlação de Pearson entre as variáveis período do curso e prevalência de cefaleia crônica não mostrou correlação significativa $(p=0,078)$.

Foram elaborados seis assertivas de acordo com os critérios da ICHD-3 (2018). As assertivas referem-se ao uso excessivo de acordo com as classes farmacológicas: analgésicos simples, anti-inflamatórios, triptanos, derivados do ergot e opioides.

Do total de 43 com critérios para cefaleia crônica, 26 (60,47\%) concordaram com as assertivas que preenchem os critérios para cefaleia por uso excessivo de medicamentos. Foi observado que a prevalência de cefaleia crônica foi uma variável dependente do consumo de alguns tipos de medicamentos, ou seja, existiu uma correlação significativa, segundo o teste de Qui-quadrado de Pearson, de ocorrência delas na amostra estudada conforme apresentado na Tabela 2. 
Tabela 2 - Associação de cefaleia crônica e uso excessivo de medicamentos, por classe farmacológica, entre os discentes de medicina.

\begin{tabular}{|c|c|c|c|c|c|c|}
\hline Medicamentos & $\begin{array}{l}\text { (n) preenchem } \\
\text { critérios para } \\
\text { cefaleia crônica } \\
\text { e utilizam a } \\
\text { classe } \\
\text { farmacológica }\end{array}$ & $\begin{array}{l}\text { (n) preenchem } \\
\text { critérios para } \\
\text { cefaleia crônica } \\
\text { e não utilizam a } \\
\text { classe } \\
\text { farmacológica }\end{array}$ & $\begin{array}{c}\text { (n) não } \\
\text { preenchem } \\
\text { critérios para } \\
\text { cefaleia } \\
\text { crônica, mas } \\
\text { utilizam a } \\
\text { classe } \\
\text { farmacológica }\end{array}$ & $\begin{array}{c}\text { (n) não } \\
\text { preenchem } \\
\text { critérios para } \\
\text { cefaleia crônica } \\
\text { e não utilizam a } \\
\text { classe } \\
\text { farmacológica }\end{array}$ & Valor de P & Significante? \\
\hline $\begin{array}{l}\text { Analgésico } \\
\text { simples }\end{array}$ & 20 & 24 & 8 & 164 & $<0.0001$ & Sim \\
\hline $\begin{array}{l}\text { Analgésico } \\
\text { combinados }\end{array}$ & 15 & 29 & 12 & 160 & $<0.0001$ & Sim \\
\hline $\begin{array}{c}\text { Derivados do } \\
\text { ergot }\end{array}$ & 9 & 35 & 4 & 168 & $<0.0001$ & Sim \\
\hline Antiinflamatórios & 2 & 42 & 2 & 170 & 0.1375 & Não \\
\hline Triptanos & 2 & 42 & 1 & 171 & 0.0450 & Sim \\
\hline Opioides & 1 & 43 & 2 & 170 & 0.5745 & Não \\
\hline
\end{tabular}

Fonte: CARNEIRO EGV, et al., 2020.

O risco para automedicação foi avaliado através de assertivas que exploram o uso de medicamentos prescritos por médicos, por conta própria, por orientação de amigos e por orientação de familiares. A maioria, $183(76,25 \%)$, discordou, totalmente ou parcialmente, da assertiva "todas as medicações que utilizo para controle da dor são prescritas por médico", sugerindo um elevado percentual de uso de medicamentos sem prescrição médica.

Destes, 173 (94,54\%) concordaram usar medicamentos para dor de cabeça por conta própria; 149 $(81,42 \%)$ por orientação de familiares não médicos e $55(30,05 \%)$ por orientação de amigos. Na amostra do estudo, $24,17 \%$ dos discentes referiram ter pai e/ou mãe da área da saúde. Apesar do elevado percentual de automedicação entre os discentes de medicina, a análise estatística do qui-quadrado de Pearson não mostrou correlação significativa entre as variáveis cefaleia crônica e automedicação $(p=0,7431)$.

Dentre os discentes que afirmam utilizar medicamentos sem prescrição médica, 57,38\% estavam nos períodos iniciais e $60,11 \%$ nos períodos mais avançados. A análise estatística da correlação de Spearman não mostrou correlação significativa entre as variáveis período do curso e automedicação $(p=0,352)$.

Os gatilhos considerados foram: período de provas/apresentação de trabalhos, qualidade do sono, tabagismo e etilismo. Os resultados encontrados mostraram que $123(51,25 \%)$ discentes $(n=123)$ referiram não ter uma qualidade do sono excelente, não se sentem bem e descansados no dia seguinte; 74 (30,83\%) demoram a dormir à noite quase todos os dias da semana; $26(10,83 \%)$ se consideraram etilistas; e $4(1,67 \%)$ se consideraram tabagista.

Ainda, 91 discentes $(37,92 \%)$ concordaram que consomem mais medicamentos para alívio da dor de cabeça no período de provas/apresentação de trabalhos, o que foi corroborado pela estatística do Quiquadrado de Pearson com correlação significativa entre as variáveis cefaleia crônica e período de provas ( $p$ $<0,0001)$. Não foi encontrada correlação significativa entre as variáveis: cefaleia crônica e insônia ( $p=$ $0,0795)$, cefaleia e etilismo $(p=0,6530)$ e cefaleia e tabagismo $(p=0,8193)$.

Dentre os fatores protetores para o uso excessivo de medicamento, nos $43 \mathrm{com}$ critérios para cefaleia crônica, 10 (23,26\%) concordaram fazer terapia não medicamentosa para alívio da dor (compressa gelada, acupuntura, psicoterapia, yoga etc.) e $22(51,16 \%)$ concordaram praticar atividade física três vezes na semana por no mínimo 30 minutos. 
A estatística analítica não encontrou correlação significativa entre as variáveis cefaleia crônica e exercício físico $(p=0,4831)$ e cefaleia crônica e terapia não medicamentosa $(p=0,1608)$. A maioria dos discentes, 198 $(82,50 \%)$, concordou com a assertiva "Estou ciente das repercussões sistêmicas que o uso excessivo de medicamentos para dor pode desencadear". Destes, 91 (45,96\%) estão nos períodos iniciais e 107 (54,04\%) nos períodos finais.

Uma quantidade expressiva de discentes, 165 (68,75\%), concordou, totalmente ou parcialmente, com a assertiva "Eu sei que o uso excessivo de medicamentos pode agravar a dor de cabeça", sendo que 73 $(44,24 \%)$ estavam nos períodos iniciais (1-4º) e 92 (55,76\%) nos períodos mais avançados (5-8ำ).

A estimativa da correlação de Pearson entre as variáveis período do curso e conhecimento das complicações mostrou que existe uma correlação positiva fraca $(p=0,003)$, ou seja, os valores associados às variáveis tendem a aumentar de forma conjunta (coeficiente de correlação $=0,203$ ).

\section{DISCUSSÃO}

Os resultados do estudo sugerem que existe uma alta prevalência $(23,50 \%)$ de cefaleia crônica entre os discentes do curso de medicina do Centro Universitário analisado. Um estudo realizado na Nigéria encontrou que $3,5 \%$ dos estudantes universitários apresentavam cefaleia crônica, com dor quase diariamente (SANYA EO, et al., 2017).

Assim como Piazza F, et al. (2012) encontraram que 9,3\% das crianças e adolescentes em idade escolar apresentam cefaleia por uso excessivo de medicamentos (CEM), alertando para a ocorrência de cefaleia crônica desde a idade escolar.

Existe uma carência na literatura mundial de estudos de prevalência da cefaleia crônica e da CEM entre os discentes de medicina. Houve uma maior prevalência de cefaleia crônica no sexo feminino $(76,74 \%)$ e nos períodos do $1^{\circ}$ ao $4^{\circ}(55,81 \%)$.

Estudo realizado na China encontrou resultado semelhante quanto ao sexo, onde a prevalência de cefaleia entre os discentes de medicina da Universidade de Soochow foi de $9,84 \%$ no sexo feminino e $4,64 \%$ no sexo masculino, e um dos motivos para a maior prevalência de cefaleia nas mulheres é o efeito dos níveis hormonais, particularmente o estrogênio (GU X e XIE YJ, 2018; LOPES DC, et al., 2015; DESOUKY DE, et al., 2019). Além disso, segundo Gu X e Xie YJ (2018) houve maior prevalência de cefaleia entre os alunos do primeiro e segundo anos quando comparado aos alunos mais velhos.

Um argumento possível para esse achado é que os alunos dos primeiros períodos sofrem mais com a adaptação ao curso (longas horas de estudo, hábito alimentar inadequado, padrão de sono irregular, privação de atividades físicas e de lazer), o que leva à elevação do nível de estresse, sendo um gatilho potencial para as cefaleias primárias (LOPES DC, et al., 2015; LEBEDEVA ER, et al., 2017).

Outro argumento é que os alunos dos últimos anos, por já terem conhecimento sobre as cefaleias, conduzam mudanças em seu estilo de vida para evitar tais gatilhos e tenham maior consciência sobre os riscos da automedicação.

O presente estudo revelou um percentual elevado de automedicação $(76,25 \%)$, achado semelhante ao encontrado no estudo de Santos R, et al. (2019), onde a automedicação estava presente em $76,50 \%$ dos discentes de medicina de uma Universidade Pública de Alagoas.

Essa elevada taxa de automedicação entre os discentes pode ser gerada tanto pelo fácil acesso aos medicamentos, como pelo fato de que essa população tem maior conhecimento acerca das patologias e seus tratamentos (AHMADI SM, et al., 2016; DESOUKY DE, et al, 2019; DA SILVA MGC, et al., 2012; SANTOS R, et al., 2019).

Segundo Badiger S, et al. (2012), os discentes utilizam esse conhecimento em 50\% do tempo para automedicação. Além disso, o fato de possuir pai/mãe da área da saúde foi associado a uma maior influência para a automedicação devido a maior facilidade na obtenção dos medicamentos (AHMADI SM, et al., 2016). 
Quanto ao conhecimento sobre as repercussões sistêmicas do uso excessivo de medicamentos, incluindo a piora da cefaleia, a maioria $(82,50 \%)$ dos discentes concordaram com a assertiva. Desses, houve maior prevalência da consciência das repercussões geradas pela prática da automedicação entre os discentes dos períodos mais avançados (54,04\%), em comparação aos dos períodos iniciais $(45,96 \%)$.

A conclusão do estudo é de que a obtenção de conhecimento médico ao longo do curso aumentou a conscientização acerca dos riscos do uso de medicações por conta própria, o que resulta na prática de automedicação responsável.

No entanto, o estudo realizado por Da Silva MGC, et al. (2012) em uma Universidade do Rio Grande do Sul encontrou dados divergentes quanto ao conhecimento (15,5\%), mas concordantes quanto à automedicação $(86,4 \%)$.

Sabe-se que a prática da automedicação está relacionada ao desenvolvimento da CEM (WESTERGAARD ML, et al., 2016). Na população em estudo, um grande percentual $(60,47 \%)$ dos discentes com cefaleia crônica apresentam critérios para a CEM apresentando correlação significativa com as classes dos analgésicos simples, analgésicos combinados, triptanos e derivados do ergot.

Alguns estudos revelam que os analgésicos simples são a principal classe de automedicação utilizada entre os discentes de medicina (ALBUSALIH F, et al., 2017; SANTOS R, et al., 2019). O trabalho de Lebedeva $E R$, et al. (2015) encontrou uma maior prevalência de CEM nos estudantes (3,1\%) quando comparado à população de trabalhadores em geral $(0,4 \%)$.

Apesar da falta de estudos na literatura mundial envolvendo a população dos discentes de medicina, o estudo de Deleu D, et al. (2001) enfatiza que é provável que o uso excessivo de analgésicos coexista com a cefaleia em estudantes de medicina, já que a grande maioria deles utiliza medicamentos sem prescrição médica.

O estudo concluiu que o principal fator desencadeante de cefaleia nos discentes foi a má qualidade do sono (51,25\%), avaliada como gatilho para a cefaleia (LOPES DC, et al., 2015; FERNÁNDEZ-DE-LAS-PEÑAS $C$, et al., 2018). Alguns trabalhos encontraram resultados semelhantes, com $92,31 \%$ dos discentes referindo falta de sono e 82,7\% sono inadequado (GU X e XIE YJ, 2018; SANTOS R, et al., 2019).

Apesar de, no presente estudo, não ter sido encontrada uma correlação estatística entre a qualidade do sono e a cefaleia crônica, entende-se que as alterações do sono podem estar associadas à rotina exaustiva, maus hábitos de vida, carga horária extensa e atividades extracurriculares, as quais agem como gatilho para a crise de cefaleia (LOPES DC, et al., 2015; IBRAHIM NK, et al., 2017).

O segundo gatilho de cefaleia avaliado nos discentes foi o período das provas e/ou apresentações/entrega de trabalhos (37,92\%). Resultado em concordância com o estudo de Ibrahim NK, et al. (2017), onde 82,6\% dos discentes referiram maior estresse no período de provas, e com o estudo de Gu X e Xie YJ (2018), onde $93,59 \%$ relacionaram como gatilho o estresse no momento de estudos. Acredita-se que nesses períodos existe uma elevação dos níveis de estresse devido à sobrecarga gerada, o que configura gatilho para a cefaleia (ICHD-3, 2018; GU X e XIE YJ, 2018).

O tabagismo foi o fator desencadeante que obteve a menor porcentagem $(1,67 \%)$ de prevalência entre os discentes do estudo. Esse dado é inferior aos achados por Desouky DE, et al. (2019) com prevalência de 4,1\% e Da Silva MGC, et al. (2012) de 10\%. Tal fato pode ser justificado pela conscientização dos discentes e a busca por hábitos de vida cada vez mais saudáveis (LOPES DC, et al., 2015).

Apenas 10,83\% dos graduandos consideraram-se etilistas, o que está em discordância com os achados na literatura, onde $64,4 \%$ e $76 \%$ dos discentes do curso de medicina se consideraram etilistas (BARBOSA FL, et al., 2013; MACHADO JNS, et al., 2016). Infere-se que pode ter ocorrido má interpretação da assertiva como possível viés, pois o termo "etilista" utilizado ter sido confundido com "alcoólatra", ou ainda, constrangimento no momento de responder à questão.

Os fatores considerados protetores entre os indivíduos com cefaleia são: prática regular de atividade física e uso de terapias não medicamentosas na fase aguda da dor como acupuntura e compressa com gelo (RUDRA RT, et al., 2016). 
Foi observado que a minoria $(23,26 \%)$ entre os discentes com critérios para cefaleia crônica, realiza terapia não medicamentosa, o que destaca a falta de conhecimento sobre os métodos alternativos e menos invasivos de tratamento; e cerca da metade $(51,16 \%)$ pratica atividade física três vezes na semana por no mínimo 30 minutos.

\section{CONCLUSÃO}

A CEM é uma patologia ainda subdiagnosticada, principalmente entre os discentes de medicina. Por isso, a elevada taxa de automedicação, a alta prevalência de cefaleia e as lacunas no conhecimento adquirido durante o curso, parecem contribuir para um cenário de cefaleia crônica com prejuízo gradual na funcionalidade e rendimento escolar dos discentes. Dessa forma, a obtenção dos medicamentos, sem prescrição médica, deve ser reavaliada pelas autoridades reguladoras a fim de minimizar os possíveis prejuízos advindos da utilização indiscriminada, assim como, práticas de conscientização sobre os riscos da automedicação e do uso crônico dessas medicações devem ser implementadas nas instituições de ensino superior. No entanto, outros estudos são necessários para aprofundar o conhecimento acerca da prevalência e do impacto da CEM entre os discentes de medicina.

\section{AGRADECIMENTOS}

Manifestamos nossos agradecimentos, em especial, ao analista de dados Arthur Renato Machado Pimentel pela contribuição na elaboração e conferência de parte dos cálculos estatísticos. E também agradecemos aos colegas que auxiliaram na revisão gramatical de nosso trabalho.

\section{REFERÊNCIAS}

1. ABAY SM, AMELO W. Assessment of self-medication practices among medical, pharmacy, and health science students in Gondar University, Ethiopia. J Young Pharm, 2010; 2(3):306-10.

2. ABDI A, et al. Prevalence of self-medication practice among health sciences students in Kermanshah, Iran. BMC Pharmacol Toxicol, 2018;19(1):1-7.

3. AHMADI SM, et al. The prevalence and affecting factors on self-medication among students of Kermanshah University of Medical Science in 2014. J Clin Diagnostic Res, 2016; 10(5):1-4.

4. ALBUSALIH F, et al. Prevalence of Self-Medication among Students of Pharmacy and Medicine Colleges of a Public Sector University in Dammam City, Saudi Arabia. Pharmacy, 2017; 5(4):1-13.

5. ALMEIDA CM, et al. Headache-related disability among medical students in Amazon: a cross-sectional study. Arq Neuropsiquiatr, 2015; 73(12):1009-13.

6. ALSHOGRAN OY, et al. Patterns of self-medication among medical and nonmedical university students in Jordan. Risk Manag Healthc Policy, 2018;11:169-76.

7. BADIGER S, et al. Self-medication patterns among medical students in South India. Australas Med J, 2012; 5(4):21720.

8. BARBOSA FL, et al. Uso de álcool entre estudantes de medicina da Universidade Federal do Maranhão. Rev Bras Educ Med, 2013; 37(1):89-95.

9. BARDIN L. Análise de Conteúdo. Lisboa: Edições 70; 1977. 220 p.

10. BIRRU EM, et al. Management of headache and associated factors among undergraduate medicine and health science students of University of Gondar, North West Ethiopia. J Headache Pain, 2016;17(1):1-9.

11. DA SILVA MGC, et al. Self-medication in university students from the city of Rio Grande, Brazil. BMC Public Health, 2012;12(1):1-7.

12. DELEU D, et al. Prevalence and Clinical Characteristics of Headache in Medical Students in Oman. Headache, 2001; 29(41):798-804.

13. DESOUKY DE, et al. Migraine, tension-type headache, and depression among Saudi female students in Taif University. J Egypt Public Health Assoc, 2019; 94(1): 1-9.

14. DINIZ BS, et al. Migrânea e rendimento escolar entre alunos de medicina. Headache Med. 2011;2(1):17-24.

15. FERNÁNDEZ-DE-LAS-PEÑAS C, et al. Sleep disturbances in tension-type headache and migraine. Ther Adv Neurol Disord, 2018;11:1-6.

16. GU X, XIE YJ. Migraine attacks among medical students in Soochow university, southeast China: A cross-sectional study. J Pain Res, 2018;11:771-81.

17. HEADACHE CLASSIFICATION COMMITTEE OF THE INTERNATIONAL HEADACHE SOCIETY (IHS). The International Classification of Headache Disorders, 3rd edition. Cephalalgia, 2018; 38(1):1-211.

18. IBRAHIM NK, et al. Prevalence, predictors and triggers of migraine headache among medical students and interns in. Pak J Med Sci, 2017; 33(2):270-5. 
19. LAI JTF, et al. Should we educate about the risks of medication overuse headache? J Headache Pain, 2014;15(1):15 .

20. LEBEDEVA ER, et al. Psychosocial factors associated with migraine and tension-type headache in medical students. Cephalalgia, 2017; 37(13):1264-71.

21. LOPES DC, et al. Cefaleia e qualidade de vida na graduação de medicina. Rev Bras Neurol e Psiquiatr, 2015;19(2):84-95.

22. LUKOVIC JA, et al. Self-medication practices and risk factors for self-medication among medical students in Belgrade, Serbia. PLoS One, 2014; 9(12):1-14.

23. MACHADO JNS, et al. Consumo de álcool entre acadêmicos de medicina. Rev Bras Pesqui em Ciências da Saúde, 2016; 2(2):46-51.

24. NOOR T, et al. Frequency, character and predisposing factor of headache among students of medical college of Karachi. J Pak Med Assoc, 2016; 66(2):159-64.

25. PIAZZA F, et al. Medication overuse headache in school-aged children: More common than expected? Headache, 2012; 52(10):1506-10.

26. RUDRA RT, et al. Acupuncture in the Management of Medication Overuse and Drug-induced Aseptic Meningitis Headache: A Case Report. JAMS J Acupunct Meridian Stud, 2020; 13(2):58-60.

27. SANTOS R, et al. Prevalência de cefaleia e seus impactos em estudantes de medicina em uma universidade pública. Rev bras neurol, 2019; 55(3):5-8.

28. SANYA EO, et al. Prevalence and clinical characteristics of headaches among undergraduate students in three tertiary institutions in Ilorin, Nigeria. Niger J Clin Pract, 2017; 20(11):1411-6.

29. STOVNER LJ, et al. Global, regional, and national burden of migraine and tension-type headache, 1990-2016: a systematic analysis for the Global Burden of Disease Study 2016. Lancet Neurol, 2018;17(11):954-76.

30. WESTERGAARD ML, et al. Medication-overuse headache: a perspective review. Ther Adv Drug Saf. 2016;7(4):14758. 